Proyecciones Journal of Mathematics

Vol. 40, No 6, pp. 1587-1602, December 2021.

Universidad Católica del Norte

Antofagasta - Chile

\title{
On the domination polynomial of a digraph: a generation function approach
}

\author{
Jorge Alencar \\ Instituto Federal de Educação, C. e T. do T. M., Brazil \\ and \\ Leonardo de Lima \\ Universidade Federal do Paraná, Brazil \\ Received : January 2021. Accepted : July 2021
}

\begin{abstract}
Let $G$ be a directed graph on $n$ vertices. The domination polynomial of $G$ is the polynomial $D(G, x)=\sum_{i=0}^{n} d(G, i) x^{i}$, where $d(G, i)$ is the number of dominating sets of $G$ with $i$ vertices. In this paper, we prove that the domination polynomial of $G$ can be obtained by using an ordinary generating function. Besides, we show that our method is useful to obtain the minimum-weighted dominating set of a graph.
\end{abstract}

Keywords: Domination polynomial, generating function, digraph, minimum-weighted dominating set problem 


\section{Introduction}

Domination polynomial in undirected graphs have been extensively studied in the literature, see for instance $[1,2,3,6]$, while domination in digraphs has not gained the same amount of attention.

Let $G=(V, E)$ be a digraph with vertex set $V(G)=\{1,2, \ldots, n\}$ and arc set $E(G)$ such that each $\operatorname{arc}(u, v) \in E(G)$ is directed and $u$ is said to be a predecessor of $v, v$ is a successor of $u$, and $u$ dominates $v$. Assume that $G$ contains no loops or multiple arcs. For any vertex $v \in V$, the open in-neighborhood of $v$ is the set $N^{-}(v)=\{u \in V:(u, v) \in E\}$ and the closed in-neighborhood of $v$ is the set $N^{-}[v]=N^{-}(v) \cup\{v\}$. For a subset $S \subseteq V$, the open in-neighborhood of $S$ is $N^{-}(S)=\bigcup_{v \in S} N^{-}(v)$, and the closed in-neighborhood of $S$ is $N^{-}[S]=\bigcup_{v \in S} N^{-}[v]$. Analogously, for any vertex $v \in V$, we define the open out-neighborhood of $v$ is the set $N^{+}(v)=\{u \in V:(v, u) \in E\}$ and the closed out-neighborhood of $v$ is the set $N^{+}[v]=N^{+}(v) \cup\{v\}$. For a set $S \subseteq V$, the open outneighborhood of $S$ is $N^{+}(S)=\bigcup_{v \in S} N^{+}(v)$ and the closed out-neighborhood of $S$ is $N^{+}[S]=\bigcup_{v \in S} N^{+}[v]$. A subset $S \subseteq V$ is an in-dominating set of $G$, if $N^{-}[S]=V$, that is, every vertex in $V \backslash S$ dominates at least one vertex in $S$. The in-domination number $\gamma^{-}(G)$ is the minimum cardinality of an in-dominating set in $G$. A subset $S \subseteq V$ is a out-dominating set of $G$, if $N^{+}[S]=V$, or equivalently, every vertex in $V \backslash S$ is dominated by at least one vertex in $S$. The out-domination number $\gamma^{+}(G)$ is the minimum cardinality of an out-dominating set in $G$. A subset $S \subseteq V$ is a total indominating set of a graph $G$ with no isolated vertices, if $N^{-}(S)=V$, or equivalently, every vertex in $V$ is dominated by at least one vertex in $S$. In [4], the authors proved that the sum of the in- and out-domination is bounded above by $4 n / 3$. The total in-domination number $\gamma_{t}^{-}(G)$ is the minimum cardinality of a total in-dominating set in $G$. A subset $S \subseteq V$ is a total out-dominating set of a graph $G$ with no isolated vertices, if $N^{+}(S)=V$, or equivalently, every vertex in $V$ dominated at least one vertex in $S$. The total out-domination number $\gamma_{t}^{+}(G)$ is the minimum cardinality of a total out-dominating set in $G$. For more information on domination in digraphs we refer the reader to $[4,5]$.

Let $d^{-}(G, i)$ be the cardinality of the in-dominating sets of size $i$, and let $d^{+}(G, i)$ be the cardinality of the out-dominating sets of size $i$ of a digraph $G$. Then the in-domination polynomial $D^{-}(G, x)$ and the out-domination 
polynomial $D^{+}(G, x)$ of $G$ are defined as follows:

$$
\begin{aligned}
& D^{-}(G, x)=\sum_{i=\gamma^{-}(G)}^{n} d^{-}(G, i) x^{i}, \\
& D^{+}(G, x)=\sum_{i=\gamma^{+}(G)}^{n} d^{+}(G, i) x^{i} .
\end{aligned}
$$

Analogously, let $\mathcal{D}_{t}^{-}(G, i)$ be the family of total in-dominating sets of a graph $\mathrm{G}$ (or $\mathcal{D}_{t}^{+}(G, i)$ for total out-dominating sets) with cardinality $i$ and let $d_{t}^{-}(G, i)=\left|\mathcal{D}^{-}(G, i)\right|\left(\right.$ or $\left.d_{t}^{+}(G, i)=\left|\mathcal{D}^{+}(G, i)\right|\right)$. Then the total indomination polynomial $D^{-}(G, x)$ and the total out-domination polynomial $D^{+}(G, x)$ of $G$ are defined as

$$
\begin{aligned}
& D_{t}^{-}(G, x)=\sum_{i=\gamma^{-}(G)}^{n} d_{t}^{-}(G, i) x^{i} \\
& D_{t}^{+}(G, x)=\sum_{i=\gamma^{+}(G)}^{n} d_{t}^{+}(G, i) x^{i}
\end{aligned}
$$

We use a similar notation when $G$ is an undirected graph. In this scenario, $G$ is a particular case of a digraph such that $N^{+}(v)=N^{-}(v)$ for all $v \in V$. Thus, when $G$ is an undirected graph we will use the following notation:

$$
\begin{aligned}
& D(G, x)=D^{+}(G, x), \\
& D_{t}(G, x)=D_{t}^{+}(G, x) .
\end{aligned}
$$

In this paper, we present an extension of the domination polynomial of an undirected graph to the domination polynomials of a digraph by using an ordinary generating function. This implies that we obtained in- and out-domination polynomials of digraphs by using an ordinary generating function. Besides, we show how our approach can be extended to the Minimum-Weighted Dominating Set problem developed (see for instance, [8]) which consists of determining the dominating set of a (di)graph which has the minimum sum of its vertices weights .

The paper is organized as follows. In Section 2, we present a generating function approach to the polynomial domination of a digraph, which can be extended to an undirected graph. In Section 3, we present examples of using the generating function approach to obtain the domination polynomial for graphs and digraphs. In Section 4, we show how our approach can be useful to the Minimum-Weighted Dominating Set problem. 


\section{Main results}

Let $Z=\{1, \ldots, m\}$. To each element $i \in Z$ we are going to assign a variable $x_{i}$ and, with those variables, we are going to use the ordinary generating function $F\left(x_{1}, \ldots, x_{m}\right)$ given by

$$
F\left(x_{1}, \ldots, x_{m}\right)=\prod_{i=1}^{m}\left(1+x_{i}\right)=\sum_{S \subseteq Z}\left(\prod_{i \in S} x_{i}\right),
$$

where the term $\prod_{i \in S} x_{i}$ of $F\left(x_{1}, \ldots, x_{m}\right)$ is uniquely determined by $S \subseteq Z$. In other words, each term of $F\left(x_{1}, \ldots, x_{m}\right)$ represents a subset of $Z$. Replacing each $x_{i}$ at Equation (2.1) by $x_{i} x$, we obtain the ordinary generating function $F\left(x_{1} x, \ldots, x_{m} x\right)$ given by

$$
\begin{gathered}
F\left(x_{1} x, \ldots, x_{m} x\right)=\prod_{i=1}^{m}\left(1+x_{i} x\right), \\
F\left(x_{1} x, \ldots, x_{m} x\right)=\sum_{S \subseteq Z}\left(x^{|S|} \prod_{i \in S} x_{i}\right), \\
F\left(x_{1} x, \ldots, x_{m} x\right)=\sum_{i=0}^{m} p_{i}\left(x_{1}, \ldots, x_{m}\right) x^{i},
\end{gathered}
$$

where on each term $x^{|S|} \prod_{i \in S} x_{i}$ of (2.3), the power of the variable $x$ is the size of the subset $S$ that uniquely determines $\prod_{i \in S} x_{i}$. Therefore, each term of $p_{i}\left(x_{1}, \ldots, x_{m}\right), i=1, \ldots, m$, is uniquely determined by a subset with exactly $i$ elements and, consequently, $p_{i}\left(x_{1}, \ldots, x_{m}\right)$ is uniquely determined by all subsets of $Z$ with exactly $i$ elements. Thus, setting $x_{i}=1$, for $i=1, \ldots, m$, we get that, $p_{i}(1, \ldots, 1)$ is the number of the subsets of $Z$ with exactly $i$ elements. From now on, we are going to use the following notation

$$
F\left(x, x_{1}, \ldots, x_{m}\right)=F\left(x_{1} x, \ldots, x_{m} x\right) .
$$

Manipulating the generating function $F\left(x, x_{1}, \ldots, x_{m}\right)$ we attempt to find directly the domination polynomials of $G$, i.e., the in- and out-domination polynomials and total in- and total out-domination polynomials. Let both $\mathbf{x}=\left(x_{1}, \ldots, x_{n}\right)$ and $\mathbf{y}=\left(y_{1}, \ldots, y_{n}\right)$ be vectors of the order of the graph $G$ such that each $x_{i}$ and $y_{i}$ are variables assigned to every vertex $i \in V(G)$. Next, we prove that the out-domination polynomial can be obtained by manipulating the generating function of (2.4). 
Theorem 1. Let $G$ be a digraph of order $n$. Then, using the following expression

$$
\int_{0}^{1} \ldots \int_{0}^{1} \frac{\partial^{n} f}{\partial y_{1} \partial y_{2} \ldots \partial y_{n}}(x, \mathbf{x}, \mathbf{y}) d y_{1} d y_{2} \ldots d y_{n}
$$

we obtain that:

(a) the out-domination polynomial of $G$ if

$$
f(x, \mathbf{x}, \mathbf{y})=\mathcal{F}^{+}(x, \mathbf{x}, \mathbf{y})=F\left(x, x_{1}\left(\prod_{j \in N^{+}[1]} y_{j}\right), \ldots, x_{n}\left(\prod_{j \in N^{+}[n]} y_{j}\right)\right) ;
$$

(b) the total out-domination polynomial of $G$ if

$$
f(x, \mathbf{x}, \mathbf{y})=\mathcal{F}_{t}^{+}(x, \mathbf{x}, \mathbf{y})=F\left(x, x_{1}\left(\prod_{j \in N^{+}(1)} y_{j}\right), \ldots, x_{n}\left(\prod_{j \in N^{+}(n)} y_{j}\right)\right) ;
$$

(c) the in-domination polynomial of $G$ if

$$
f(x, \mathbf{x}, \mathbf{y})=\mathcal{F}^{-}(x, \mathbf{x}, \mathbf{y})=F\left(x, x_{1}\left(\prod_{j \in N^{-}[1]} y_{j}\right), \ldots, x_{n}\left(\prod_{j \in N^{-}[n]} y_{j}\right)\right)
$$

(d) the total in-domination polynomial of $G$ if

$$
f(x, \mathbf{x}, \mathbf{y})=\mathcal{F}_{t}^{-}(x, \mathbf{x}, \mathbf{y})=F\left(x, x_{1}\left(\prod_{j \in N^{-}(1)} y_{j}\right), \ldots, x_{n}\left(\prod_{j \in N^{-}(n)} y_{j}\right)\right)
$$

Proof. For each $i=1,2, \ldots, n$, consider replacing $x_{i}$ in Equation (2.5) by

$$
x_{i}\left(\prod_{j \in N^{+}[i]} y_{i}\right) .
$$

Thus, we obtain the following generating function

$$
\begin{aligned}
\mathcal{F}^{+}(x, \mathbf{x}, \mathbf{y}) & =F\left(x, x_{1}\left(\prod_{j \in N^{+}[1]} y_{j}\right), \ldots, x_{n}\left(\prod_{j \in N^{+}[n]} y_{j}\right)\right) \\
& =\prod_{i=1}^{n}\left(1+x x_{i}\left(\prod_{j \in N^{+}[i]} y_{j}\right)\right) \\
& =\sum_{S \subseteq V(G)}\left(x^{|S|} \prod_{i \in S} x_{i}\left(\prod_{j \in N^{+}[i]} y_{j}\right)\right) \\
& =\sum_{i=0}^{n} p_{i}(\mathbf{x}, \mathbf{y}) x^{i} .
\end{aligned}
$$


For each contribution of $p_{i}(\mathbf{x}, \mathbf{y})$ there exist subsets of vertices $\bar{Y}, \bar{X} \subseteq$ $V=\{1,2, \ldots, n\}$ such that this contribution is equal to

$$
\prod_{k \in \bar{X}} x_{k} \prod_{j \in \bar{Y}} y_{j}^{t_{j}}
$$

where $t_{j}$ is the number of vertices in $\bar{X}$ such that $j$ are in its closed outneighbourhood, and $|\bar{X}|=i$. Thus, $\bar{Y}$ represents the vertices that belongs to the subdigraph of $G$ induced by the closed out-neighbourhood of the vertices in $\bar{X}$. In particular, if $\bar{Y}=V$ the subdigraph obtained is $G$ itself, and in this case we have that $\bar{X}$ is an out-dominating set, and, reciprocally, if $\bar{X}$ is an out-dominating set then $\bar{Y}=V$. Therefore, when we consider

$$
\mathcal{H}^{+}(x, \mathbf{x})=\int_{0}^{1} \ldots \int_{0}^{1} \frac{\partial^{n} \mathcal{F}^{+}}{\partial y_{1} \partial y_{2} \ldots \partial y_{n}}(x, \mathbf{x}, \mathbf{y}) d y_{1} d y_{2} \ldots d y_{n}=\sum_{i=0}^{n} q_{i}(\mathbf{x}) x^{i}
$$

we have that $q_{i}(\mathbf{x})$ lists the out-domination sets of $G$ with exactly $i$ points, i.e., if $\mathbf{x}=\mathbf{1}_{n}$, where $\mathbf{1}_{n}$ is the $n$-dimensional vector of all ones, we get

$$
D^{+}(G, x)=\mathcal{H}^{+}\left(x, \mathbf{1}_{n}\right) .
$$

From the fundamental theorem of calculus, we can rewrite $\mathcal{H}^{+}$as follows

$$
\mathcal{H}^{+}(x, \mathbf{x})=\left.\left.\left.\left(\mathcal{F}^{+}(x, \mathbf{x}, \mathbf{y})\right)\right|_{y_{1}=0} ^{1}\right|_{y_{2}=0} ^{1} \ldots\right|_{y_{n}=0} ^{1}
$$

or, equivalently,

$$
\mathcal{H}^{+}(x, \mathbf{x})=\sum_{v \in\{0,1\}^{n}}(-1)^{n-v^{\top} \mathbf{1}_{n}} \mathcal{F}^{+}(x, \mathbf{x}, v)
$$

where $v^{\top} \mathbf{1}_{n}$ represents the inner product between vectors $\mathbf{1}_{n}$ and $v$. The proof of item (a) is complete.

For items (b), (c) and (d), we can use the same idea to the generating functions below: 


$$
\begin{aligned}
& \mathcal{F}^{-}(x, \mathbf{x}, \mathbf{y})=\prod_{i=1}^{n}\left(1+x x_{i}\left(\prod_{j \in N^{-}[i]} y_{j}\right)\right), \\
& \mathcal{F}_{t}^{+}(x, \mathbf{x}, \mathbf{y})=\prod_{i=1}^{n}\left(1+x x_{i}\left(\prod_{j \in N^{+}(i)} y_{j}\right)\right), \\
& \mathcal{F}_{t}^{-}(x, \mathbf{x}, \mathbf{y})=\prod_{i=1}^{n}\left(1+x x_{i}\left(\prod_{j \in N^{-}(i)} y_{j}\right)\right),
\end{aligned}
$$

and we obtain $D^{-}(G, x), D_{t}^{+}(G, x)$ and $D_{t}^{-}(G, x)$, respectively.

In particular, if $G$ is undirected we will use the following notation:

$$
\begin{array}{ll}
\mathcal{F}(x, \mathbf{x}, \mathbf{y}) & =\mathcal{F}^{+}(x, \mathbf{x}, \mathbf{y}), \\
\mathcal{H}(x, \mathbf{x}) & =\mathcal{H}^{+}(x, \mathbf{x}), \\
\mathcal{F}_{t}(x, \mathbf{x}, \mathbf{y}) & =\mathcal{F}_{t}^{+}(x, \mathbf{x}, \mathbf{y}), \\
\mathcal{H}_{t}(x, \mathbf{x}) & =\mathcal{H}_{t}^{+}(x, \mathbf{x}) .
\end{array}
$$

In the next section examples are explored to show how our method works for undirected and directed graphs.

\section{Undirected and directed graph examples}

In order to show our method, consider the graph $G=(V, E)$ with vertex set given by $V=\{1,2,3,4,5,6\}$ and edge set

$E=\{\{1,2\},\{1,3\},\{1,4\},\{2,3\},\{4,5\},\{4,6\},\{5,6\}\}$, as it is shown by Figure 1. At first, we consider the undirected case of the graph, after that we will put a orientation on its edges in order to obtain a digraph to show a more general example. To construct the generating functions mentioned in the previous section we will represent the $i$-th vertex, $i \in\{1,2,3,4,5,6\}$, in $V$ using the variables $x_{i}$ and $y_{i}$.

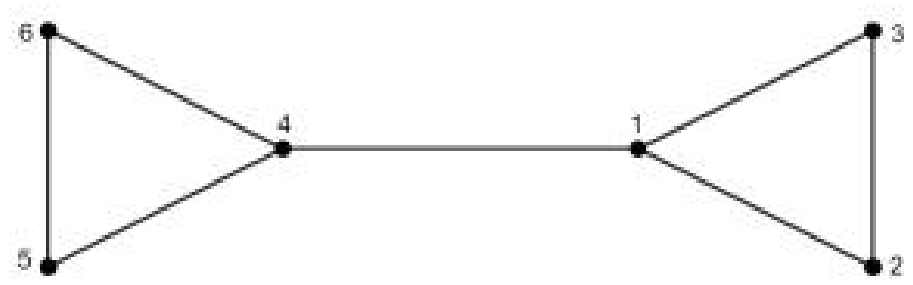

Figure 1: Example of an undirected graph $G$. 


\subsection{When $G$ is an undirected graph}

In this case we will consider the graph of the Figure 1. We use variables $x_{i}$ and $y_{j}$ with $i, j \in\{1,2,3,4,5,6\}$ to represent the vertices in $V$, and get the following generating function:

$$
\begin{aligned}
\mathcal{F}_{t}(x, \mathbf{x}, \mathbf{y})= & \left(1+x x_{1} y_{2} y_{3} y_{4}\right)\left(1+x x_{2} y_{1} y_{3}\right) \\
& \left(1+x x_{3} y_{1} y_{2}\right)\left(1+x x_{4} y_{1} y_{5} y_{6}\right) \\
& \left(1+x x_{5} y_{4} y_{6}\right)\left(1+x x_{6} y_{4} y_{5}\right) \\
= & \sum_{i=0}^{6} p_{i}(\mathbf{x}, \mathbf{y}) x^{i},
\end{aligned}
$$

where $\mathbf{x}=\left(x_{1}, x_{2}, x_{3}, x_{4}, x_{5}, x_{6}\right)$ and $\mathbf{y}=\left(y_{1}, y_{2}, y_{3}, y_{4}, y_{5}, y_{6}\right)$. Note that the $x^{i}$ coefficients given by $p_{i}(\mathbf{x}, \mathbf{y})$ are equal to

$$
\prod_{k \in \bar{X}} x_{k} \prod_{j \in \bar{Y}} y_{j}^{p_{j}},
$$

where $\bar{X}$ is a subset of $\{1,2,3,4,5,6\}, \bar{Y}$ is the set of all vertices that are in the neighborhood of $\bar{X}$, and $p_{j}$ is the degree of vertex $j$ in the subgraph induced by the vertices in $\bar{X} \cup N^{+}(\bar{X})$. Notice that $\bar{Y}=V$ if and only if $\bar{X}$ is a total dominating set of $G$. If we derive the generating function $\mathcal{F}_{t}$ in terms of the variables $y_{1}, y_{2}, y_{3}, y_{4}, y_{5}$ and $y_{6}$, any contribution in $p_{i}(\mathbf{x}, \mathbf{y})$ will be zero if it has not all $y_{i}$ 's, $i \in\{1,2,3,4,5,6\}$, that is, the only contributions that will not be zero by the derivation are the ones which represent a total dominating set. Because of the derivation we will obtain some unnecessary coefficients and the variables $y_{i}$ 's, $i \in\{1,2,3,4,5,6\}$ are unnecessary as well. To solve this problem, we integrate the obtained function from 0 to 1 in $y_{i}$ 's, $i \in\{1,2,3,4,5,6\}$. Formally, we obtain the following generating function

$$
\begin{aligned}
\mathcal{H}_{t}(x, \mathbf{x})= & \int_{0}^{1} \ldots \int_{0}^{1} \frac{\partial^{6} F_{t}}{\partial y_{1} \partial y_{2} \ldots \partial y_{6}}(x, \mathbf{x}, \mathbf{y}) d y_{1} d y_{2} \ldots d y_{6} \\
= & x^{2} x_{1} x_{4}+x^{3} x_{1} x_{2} x_{4}+x^{3} x_{1} x_{3} x_{4}+x^{4} x_{1} x_{2} x_{3} x_{4} \\
& +x^{3} x_{1} x_{4} x_{5}+x^{4} x_{1} x_{2} x_{4} x_{5}+x^{4} x_{1} x_{3} x_{4} x_{5}+x^{4} x_{2} x_{3} x_{4} x_{5} \\
& +x^{5} x_{1} x_{2} x_{3} x_{4} x_{5}+x^{3} x_{1} x_{4} x_{6}+x^{4} x_{1} x_{2} x_{4} x_{6} \\
& +x^{4} x_{1} x_{3} x_{4} x_{6}+x^{4} x_{2} x_{3} x_{4} x_{6}+x^{5} x_{1} x_{2} x_{3} x_{4} x_{6} \\
& +x^{4} x_{1} x_{2} x_{5} x_{6}+x^{4} x_{1} x_{3} x_{5} x_{6}+x^{4} x_{2} x_{3} x_{5} x_{6} \\
& +x^{5} x_{1} x_{2} x_{3} x_{5} x_{6}+x^{4} x_{1} x_{4} x_{5} x_{6}+x^{5} x_{1} x_{2} x_{4} x_{5} x_{6} \\
& +x^{5} x_{1} x_{3} x_{4} x_{5} x_{6}+x^{5} x_{2} x_{3} x_{4} x_{5} x_{6}+x^{6} x_{1} x_{2} x_{3} x_{4} x_{5} x_{6} \\
= & \sum_{i=0}^{6} q_{i}(\mathbf{x}) x^{i}
\end{aligned}
$$


Since each contribution of $q_{i}(\mathbf{x})$ represents a total dominating set, when we apply $\mathbf{x}=\mathbf{1}_{n}$ in $\mathcal{H}_{t}(x, X)$, we obtain the total domination polynomial:

$$
D_{t}(G, x)=x^{2}+4 x^{3}+11 x^{4}+6 x^{5}+x^{6} .
$$

Therefore, the total domination number of $G$ is 2 , and the coefficient of $x^{2}$ in $\mathcal{H}_{t}(x, \mathbf{x})$ is given by $x_{1} x_{4}$ and it represents all the total dominating sets with 2 elements.

Now, using the generating function $\mathcal{F}(x, \mathbf{x}, \mathbf{y})=\mathcal{F}^{+}(x, \mathbf{x}, \mathbf{y})$, we get the following:

$$
\begin{aligned}
\mathcal{F}(x, \mathbf{x}, \mathbf{y})= & \left(1+x x_{1} y_{1} y_{2} y_{3} y_{4}\right)\left(1+x x_{2} y_{2} y_{1} y_{3}\right) \\
& \left(1+x x_{3} y_{3} y_{1} y_{2}\right)\left(1+x x_{4} y_{4} y_{1} y_{5} y_{6}\right) \\
& \left(1+x x_{5} y_{5} y_{4} y_{6}\right)\left(1+x x_{6} y_{6} y_{4} y_{5}\right) \\
= & \sum_{i=0}^{6} p_{i}(\mathbf{x}, \mathbf{y}) x^{i}
\end{aligned}
$$

We have that for each contribution $p_{i}(\mathbf{x}, \mathbf{y})$ there exists subsets of vertices $\bar{Y}, \bar{X} \subseteq\{1,2,3,4,5,6\}=V$ such that this term is equals to

$$
\prod_{k \in \bar{X}} x_{k} \prod_{j \in \bar{Y}} y_{j}^{p_{j}},
$$

where $p_{j}$ is the number of vertices in $\bar{X}$ such that $j$ are in its closed neighbourhood, and $|\bar{X}|=i$. So, $\bar{Y}=V$ if and only if $\bar{X}$ is a dominating set of $G$. By applying the same procedure used before, we obtain the generating function

$$
\begin{aligned}
\mathcal{H}(x, \mathbf{x})= & x^{2} x_{1} x_{4}+x^{2} x_{2} x_{4}+x^{3} x_{1} x_{2} x_{4}+x^{2} x_{3} x_{4}+x^{3} x_{1} x_{3} x_{4}+x^{3} x_{2} x_{3} x_{4} \\
& +x^{4} x_{1} x_{2} x_{3} x_{4}+x^{2} x_{1} x_{5}+x^{2} x_{2} x_{5}+x^{3} x_{1} x_{2} x_{5}+x^{2} x_{3} x_{5}+x^{3} x_{1} x_{3} x_{5} \\
& +x^{3} x_{2} x_{3} x_{5}+x^{4} x_{1} x_{2} x_{3} x_{5}+x^{3} x_{1} x_{4} x_{5}+x^{3} x_{2} x_{4} x_{5}+x^{4} x_{1} x_{2} x_{4} x_{5} \\
& +x^{3} x_{3} x_{4} x_{5}+x^{4} x_{1} x_{3} x_{4} x_{5}+x^{4} x_{2} x_{3} x_{4} x_{5}+x^{5} x_{1} x_{2} x_{3} x_{4} x_{5}+x^{2} x_{1} x_{6} \\
& +x^{2} x_{2} x_{6}+x^{3} x_{1} x_{2} x_{6}+x^{2} x_{3} x_{6}+x^{3} x_{1} x_{3} x_{6}+x^{3} x_{2} x_{3} x_{6}+x^{4} x_{1} x_{2} x_{3} x_{6} \\
& +x^{3} x_{1} x_{4} x_{6}+x^{3} x_{2} x_{4} x_{6}+x^{4} x_{1} x_{2} x_{4} x_{6}+x^{3} x_{3} x_{4} x_{6}+x^{4} x_{1} x_{3} x_{4} x_{6} \\
& +x^{4} x_{2} x_{3} x_{4} x_{6}+x^{5} x_{1} x_{2} x_{3} x_{4} x_{6}+x^{3} x_{1} x_{5} x_{6}+x^{3} x_{2} x_{5} x_{6}+x^{4} x_{1} x_{2} x_{5} x_{6} \\
& +x^{3} x_{3} x_{5} x_{6}+x^{4} x_{1} x_{3} x_{5} x_{6}+x^{4} x_{2} x_{3} x_{5} x_{6}+x^{5} x_{1} x_{2} x_{3} x_{5} x_{6} \\
& +x^{4} x_{1} x_{4} x_{5} x_{6}+x^{4} x_{2} x_{4} x_{5} x_{6}+x^{5} x_{1} x_{2} x_{4} x_{5} x_{6}+x^{4} x_{3} x_{4} x_{5} x_{6} \\
& +x^{5} x_{1} x_{3} x_{4} x_{5} x_{6}+x^{5} x_{2} x_{3} x_{4} x_{5} x_{6}+x^{6} x_{1} x_{2} x_{3} x_{4} x_{5} x_{6} \\
= & \sum_{i=0}^{6} q_{i}(\mathbf{x}) x^{i}
\end{aligned}
$$


where each contribution of $q_{i}(\mathbf{x})$ represents a dominating set of size $i$. Notice that the coefficient of $x^{2}$ in $\mathcal{H}_{t}(x, \mathbf{x})$ is given by

$$
x_{1} x_{4}+x_{2} x_{4}+x_{3} x_{4}+x_{1} x_{5}+x_{2} x_{5}+x_{3} x_{5}+x_{1} x_{6}+x_{2} x_{6}+x_{3} x_{6},
$$

which represents all the dominating sets with 2 elements. Taking $\mathbf{x}=\mathbf{1}_{n}$ in $\mathcal{H}(x, \mathbf{x})$, we obtain the domination polynomial:

$$
D(G, x)=9 x^{2}+18 x^{3}+15 x^{4}+6 x^{5}+x^{6} .
$$

Therefore, the domination number of $G$ is equal to 2 and the number of vertex subsets with cardinality two that are domination sets is equal to 9 .

\subsection{When $G$ is a directed graph}

In order to exemplify the technique for a digraph, we give an orientation to graph $G$, which is represented in Figure 2. In this case, $V=\{1,2,3,4,5,6\}$ and $E=\{(1,2),(2,3),(3,1),(1,4),(4,6),(6,5),(5,4)\}$.

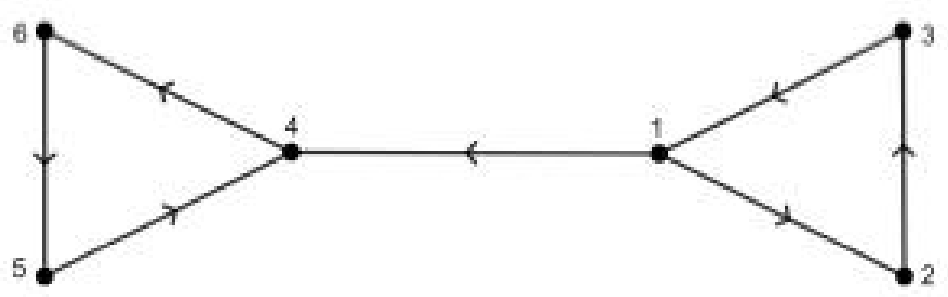

Figure 2: Directed graph $G$.

The variables $x_{i}$ and $y_{j}$, with $i, j \in\{1,2,3,4,5,6\}$, represent the vertices in $V$. We construct the functions related to the out-domination polynomial, total out-domination polynomial, in-domination polynomial, and total indomination polynomial, which we represent in the following four cases.

Case 1: To construct the out-domination polynomial $\mathcal{D}^{+}(G, x)$, we first construct the following generating function:

$$
\begin{aligned}
\mathcal{F}^{+}(x, X, Y)= & \left(1+x x_{1} y_{1} y_{2} y_{4}\right)\left(1+x x_{2} y_{2} y_{3}\right)\left(1+x x_{3} y_{1} y_{3}\right) \\
& \left(1+x x_{4} y_{4} y_{6}\right)\left(1+x x_{5} y_{4} y_{5}\right)\left(1+x x_{6} y_{5} y_{6}\right)
\end{aligned}
$$

Thus, from (2.6) we obtain 


$$
\begin{aligned}
\mathcal{H}^{+}(x, \mathbf{x})= & x^{4} x_{1} x_{2} x_{4} x_{5}+x^{4} x_{1} x_{3} x_{4} x_{5}+x^{4} x_{2} x_{3} x_{4} x_{5}+x^{5} x_{1} x_{2} x_{3} x_{4} x_{5} \\
& +x^{3} x_{1} x_{2} x_{6}+x^{3} x_{1} x_{3} x_{6}+x^{4} x_{1} x_{2} x_{3} x_{6}+x^{4} x_{1} x_{2} x_{4} x_{6} \\
& +x^{4} x_{1} x_{3} x_{4} x_{6}+x^{4} x_{2} x_{3} x_{4} x_{6}+x^{5} x_{1} x_{2} x_{3} x_{4} x_{6} \\
& +x^{4} x_{1} x_{2} x_{5} x_{6}+x^{4} x_{1} x_{3} x_{5} x_{6}+x^{4} x_{2} x_{3} x_{5} x_{6} \\
& +x^{5} x_{1} x_{2} x_{3} x_{5} x_{6}+x^{5} x_{1} x_{2} x_{4} x_{5} x_{6}+x^{5} x_{1} x_{3} x_{4} x_{5} x_{6} \\
& +x^{5} x_{2} x_{3} x_{4} x_{5} x_{6}+x^{6} x_{1} x_{2} x_{3} x_{4} x_{5} x_{6} \\
= & \sum_{i=0}^{6} q_{i}(\mathbf{x}) x^{i}
\end{aligned}
$$

where each coefficient $q_{i}(\mathbf{x})$ represents an out-dominating set. Taking $\mathbf{x}=$ $\mathbf{1}_{n}$ in $\mathcal{H}^{+}(x, \mathbf{x})$, we get the out-domination polynomial:

$$
D^{+}(G, x)=2 x^{3}+10 x^{4}+6 x^{5}+x^{6} .
$$

Therefore, the out-domination number of $G$ is equal to 3 , and the coefficient of $x^{3}$ in $\mathcal{H}^{+}(x, \mathbf{x})$ given by

$$
x_{1} x_{2} x_{6}+x_{1} x_{3} x_{6},
$$

represents all the out-dominating sets with 3 vertices.

Case 2: To construct the total out-domination polynomial $\mathcal{D}_{t}^{+}(G, x)$, we first construct the following generating function:

$$
\begin{aligned}
\mathcal{F}_{t}^{+}(x, \mathbf{x}, \mathbf{y})= & \left(1+x x_{1} y_{2} y_{4}\right)\left(1+x x_{2} y_{3}\right)\left(1+x x_{3} y_{1}\right) \\
& \left(1+x x_{4} y_{6}\right)\left(1+x x_{5} y_{4}\right)\left(1+x x_{6} y_{5}\right) .
\end{aligned}
$$

From (2.6), we obtain the generating function

$$
\mathcal{H}_{t}^{+}(x, \mathbf{x})=x^{5} x_{1} x_{2} x_{3} x_{4} x_{6}+x^{6} x_{1} x_{2} x_{3} x_{4} x_{5} x_{6} .
$$

Taking $\mathbf{x}=\mathbf{1}_{n}$ in $\mathcal{H}^{+}(x, \mathbf{x})$, we obtain the total out-domination polynomial:

$$
D^{+}(G, x)=x^{5}+x^{6},
$$

and so the total out-domination number of $G$ is equal to 5 . The coefficient of $x^{5}$ in $\mathcal{H}^{+}(x, \mathbf{x}), x_{1} x_{2} x_{3} x_{4} x_{6}$, represents all the total out-dominating sets with 5 elements.

Case 3: To construct the in-domination polynomial $\mathcal{D}^{-}(G, x)$, we first construct the following generating function:

$$
\begin{aligned}
\mathcal{F}^{-}(x, X, Y)= & \left(1+x x_{1} y_{1} y_{3}\right)\left(1+x x_{2} y_{1} y_{2}\right)\left(1+x x_{3} y_{2} y_{3}\right) \\
& \left(1+x x_{4} y_{1} y_{4} y_{5}\right)\left(1+x x_{5} y_{5} y_{6}\right)\left(1+x x_{6} y_{4} y_{6}\right) .
\end{aligned}
$$


From (2.6), we obtain

$$
\begin{aligned}
\mathcal{H}^{-}(x, \mathbf{x})= & x^{4} x_{1} x_{2} x_{4} x_{5}+x^{3} x_{3} x_{4} x_{5}+x^{4} x_{1} x_{3} x_{4} x_{5}+x^{4} x_{2} x_{3} x_{4} x_{5} \\
& +x^{5} x_{1} x_{2} x_{3} x_{4} x_{5}+x^{4} x_{1} x_{2} x_{4} x_{6}+x^{3} x_{3} x_{4} x_{6}+x^{4} x_{1} x_{3} x_{4} x_{6} \\
& +x^{4} x_{2} x_{3} x_{4} x_{6}+x^{5} x_{1} x_{2} x_{3} x_{4} x_{6}+x^{4} x_{1} x_{2} x_{5} x_{6}+x^{4} x_{1} x_{3} x_{5} x_{6} \\
& +x^{4} x_{2} x_{3} x_{5} x_{6}+x^{5} x_{1} x_{2} x_{3} x_{5} x_{6}+x^{5} x_{1} x_{2} x_{4} x_{5} x_{6} \\
& +x^{4} x_{3} x_{4} x_{5} x_{6}+x^{5} x_{1} x_{3} x_{4} x_{5} x_{6}+x^{5} x_{2} x_{3} x_{4} x_{5} x_{6} \\
& +x^{6} x_{1} x_{2} x_{3} x_{4} x_{5} x_{6} \\
= & \sum_{i=0}^{6} q_{i}(\mathbf{x}) x^{i}
\end{aligned}
$$

where each contribution of $q_{i}(\mathbf{x})$ represents an in-dominating set, then when we apply $\mathbf{x}=\mathbf{1}_{n}$ in $\mathcal{H}^{-}(x, \mathbf{x})$, we obtain the in-domination polynomial:

$$
D^{-}(G, x)=2 x^{3}+10 x^{4}+6 x^{5}+x^{6} .
$$

Therefore, the in-domination number of $G$ is 3 , and the coefficient of $x^{3}$ in $\mathcal{H}^{-}(x, \mathbf{x})$,

$$
x_{3} x_{4} x_{5}+x_{3} x_{4} x_{6},
$$

represents all the in-dominating sets with 3 vertices.

Case 4: To construct the total in-domination polynomial $\mathcal{D}_{t}^{-}(G, x)$, first we construct the following generating function:

$$
\begin{aligned}
\mathcal{F}_{t}^{-}(x, \mathbf{x}, \mathbf{y})= & \left(1+x x_{1} y_{3}\right)\left(1+x x_{2} y_{1}\right)\left(1+x x_{3} y_{2}\right) \\
& \left(1+x x_{4} y_{1} y_{5}\right)\left(1+x x_{5} y_{6}\right)\left(1+x x_{6} y_{4}\right)
\end{aligned}
$$

Thus, applying (2.6), we obtain the generating function

$$
\mathcal{H}_{t}^{-}(x, \mathbf{x})=x^{5} x_{1} x_{3} x_{4} x_{5} x_{6}+x^{6} x_{1} x_{2} x_{3} x_{4} x_{5} x_{6},
$$

then when we apply $\mathbf{x}=\mathbf{1}_{n}$ in $\mathcal{H}^{-}(x, \mathbf{x})$, we obtain the total in-domination polynomial

$$
D^{-}(G, x)=x^{5}+x^{6} \text {. }
$$

Therefore the total in-domination number of $G$ is 5 , and the coefficient of $x^{5}$ in $\mathcal{H}^{-}(x, \mathbf{x}), x_{1} x_{3} x_{4} x_{5} x_{6}$, represents the unique in-dominating set with 5 vertices of graph $G$. 


\section{An application to the Minimum-Weighted Dominating Set problem}

In this section, we apply the technique of Section 2 to the MinimumWeighted Dominating Set problem. We assume that $G=(V, E, w)$ is an undirected graph where a weight $w_{i}>0$ is assigned to each vertex $v_{i}$ for $i=1, \ldots, n$.

The Minimum-Weighted Dominating Set problem consists of finding the dominating set with smallest weight, i.e., it consists of finding the weighted dominating set for which the sum of the weights of the vertices that belongs to the set is the smallest possible. Write $W$ for the set defined as

$W=\left\{w\left(j_{1}\right)+\ldots+w\left(j_{i}\right):\left(i, j_{1}, \ldots, j_{i} \in\{1, \ldots, n\}\right) \wedge\left(j_{k} \neq j_{l}\right.\right.$ for all $\left.\left.k \neq l\right)\right\}$.

From the set $W$, we can define the following generating function

$$
\begin{aligned}
\mathcal{W}(x, \mathbf{x}, \mathbf{y}) & =\mathcal{F}\left(x, x^{w(1)-1} x_{1}\left(\prod_{j \in N[1]} y_{j}\right), \ldots, x^{w(n)-1} x_{n}\left(\prod_{j \in N[n]} y_{j}\right)\right) \\
& =\prod_{i=1}^{n}\left(1+x^{w(1)} x_{i}\left(\prod_{j \in N[i]} y_{j}\right)\right) \\
& =\sum_{i \in W} p_{i}(\mathbf{x}, \mathbf{y}) x^{i},
\end{aligned}
$$

where each coefficient $p_{i}(\mathbf{x}, \mathbf{y})$ is equal to

$$
\prod_{k \in \bar{X}} x_{k} \prod_{j \in \bar{Y}} y_{j}^{p_{j}}
$$

such that $\bar{X}, \bar{Y}$ are subsets of $V=\{1, \ldots, n\}$, and $p_{j}$ is the number of vertices in $\bar{X}$ such that $j$ is in its closed neighbourhood, $\sum_{k \in \bar{X}} w(k)=i$. So $\bar{Y}$ contains the vertices that belong to the graph $G$ induced by the closed neighbourhood of the vertices in $\bar{X}$. In particular, if $\bar{Y}=V$ the graph obtained is $G$ itself, and in this case notice that $\bar{X}$ is a weighted dominating set of weight $\sum_{k \in \bar{X}} w(k)$. Reciprocally, if $\bar{X}$ is a weighted dominating set then $\bar{Y}=V$. Therefore, by construction, when we take

$$
\mathcal{H}_{w}(x, \mathbf{x})=\int_{0}^{1} \ldots \int_{0}^{1} \frac{\partial^{n} \mathcal{W}}{\partial y_{1} \partial y_{2} \ldots \partial y_{n}}(x, \mathbf{x}, \mathbf{y}) d y_{1} d y_{2} \ldots d y_{n}=\sum_{i \in W} q_{i}(\mathbf{x}) x^{i},
$$

we have that $q_{i}(\mathbf{x})$ lists all weighted domination sets of $G$ with weight exactly equals to $i$, i.e., if $\mathbf{x}=\mathbf{1}_{n}$, where $\mathbf{1}_{n}$ is the $n$-dimensional vector of ones, then

$$
D_{w}(G, x)=\mathcal{H}\left(x, \mathbf{1}_{n}\right),
$$


where $D_{w}(G, x)$ is the weighted domination polynomial of $G$. It is easy to see that we can obtain the same equation if we construct the generating function $\mathcal{H}(x, X)$ of the graph $G$ ignoring the node weight function. In this way,

$$
\mathcal{H}_{w}(x, X)=\mathcal{H}\left(x, x^{w(1)-1} x_{1}, \ldots, x^{w(n)-1} x_{n}\right) .
$$

In order to illustrate our approach, consider the graph $G=(V, E, w)$ of the Figure 1 such that the weights are given by

$$
w(1)=7, w(2)=8, w(3)=4, w(4)=8, w(5)=6, w(6)=3 .
$$

Besides, we will use the variables $x_{i}$ and $y_{j}$, with $i, j \in\{1,2,3,4,5,6\}$, to represent the vertices in $V$ and to construct the generating function

$$
\begin{aligned}
\mathcal{W}(x, \mathbf{x}, \mathbf{y})= & \left(1+x^{7} x_{1} y_{1} y_{2} y_{3} y_{4}\right)\left(1+x^{8} x_{2} y_{1} y_{2} y_{3}\right)\left(1+x^{4} x_{3} y_{1} y_{2} y_{3}\right) \\
= & \left(1+x^{8} x_{4} y_{1} y_{4} y_{5} y_{6}\right)\left(1+x^{6} x_{5} y_{4} y_{5} y_{6}\right)\left(1+x^{3} x_{6} y_{4} y_{5} y_{6}\right) \\
& \sum_{i \in W} p_{i}(\mathbf{x}, \mathbf{y}) x^{i} .
\end{aligned}
$$

By applying (2.6), we obtain the generating function

$$
\begin{aligned}
\mathcal{H}_{w}(x, \mathbf{x})= & x^{15} x_{1} x_{4}+x^{16} x_{2} x_{4}+x^{23} x_{1} x_{2} x_{4}+x^{12} x_{3} x_{4}+x^{19} x_{1} x_{3} x_{4} \\
& +x^{20} x_{2} x_{3} x_{4}+x^{27} x_{1} x_{2} x_{3} x_{4}+x^{13} x_{1} x_{5}+x^{14} x_{2} x_{5}+x^{21} x_{1} x_{2} x_{5} \\
& +x^{10} x_{3} x_{5}+x^{17} x_{1} x_{3} x_{5}+x^{18} x_{2} x_{3} x_{5}+x^{25} x_{1} x_{2} x_{3} x_{5}+ \\
& x^{21} x_{1} x_{4} x_{5}+x^{22} x_{2} x_{4} x_{5}+x^{29} x_{1} x_{2} x_{4} x_{5}+x^{18} x_{3} x_{4} x_{5} \\
& +x^{25} x_{1} x_{3} x_{4} x_{5}+x^{26} x_{2} x_{3} x_{4} x_{5}+x^{33} x_{1} x_{2} x_{3} x_{4} x_{5} \\
& +x^{10} x_{1} x_{6}+x^{11} x_{2} x_{6}+x^{18} x_{1} x_{2} x_{6}+x^{7} x_{3} x_{6} \\
& +x^{14} x_{1} x_{3} x_{6}+x^{15} x_{2} x_{3} x_{6}+x^{22} x_{1} x_{2} x_{3} x_{6}+x^{18} x_{1} x_{4} x_{6} \\
& +x^{19} x_{2} x_{4} x_{6}+x^{26} x_{1} x_{2} x_{4} x_{6}+x^{15} x_{3} x_{4} x_{6}+x^{22} x_{1} x_{3} x_{4} x_{6}+ \\
& x^{23} x_{2} x_{3} x_{4} x_{6}+x^{30} x_{1} x_{2} x_{3} x_{4} x_{6}+x^{16} x_{1} x_{5} x_{6}+x^{17} x_{2} x_{5} x_{6} \\
& +x^{24} x_{1} x_{2} x_{5} x_{6}+x^{13} x_{3} x_{5} x_{6}+x^{20} x_{1} x_{3} x_{5} x_{6}+x^{21} x_{2} x_{3} x_{5} x_{6} \\
& +x^{28} x_{1} x_{2} x_{3} x_{5} x_{6}+x^{24} x_{1} x_{4} x_{5} x_{6}+x^{25} x_{2} x_{4} x_{5} x_{6}+ \\
& x^{32} x_{1} x_{2} x_{4} x_{5} x_{6}+x^{21} x_{3} x_{4} x_{5} x_{6}+x^{28} x_{1} x_{3} x_{4} x_{5} x_{6} \\
& +x^{29} x_{2} x_{3} x_{4} x_{5} x_{6}+x^{36} x_{1} x_{2} x_{3} x_{4} x_{5} x_{6} \\
= & \sum_{i \in W} q_{i}(\mathbf{x}) x^{i}
\end{aligned}
$$

where each contribution of $q_{i}(\mathbf{x})$ represents a weighted dominating set with weight exactly equal to $i$. Taking $\mathbf{x}=\mathbf{1}_{n}$ in $\mathcal{H}(x, \mathbf{x})$, we obtain the weighted domination polynomial

$$
\begin{aligned}
D_{w}(G, x)= & x^{7}+2 x^{10}+x^{11}+x^{12}+2 x^{13}+2 x^{14}+3 x^{15}+2 x^{16} \\
& +2 x^{17}+4 x^{18}+2 x^{19}+2 x^{20}+4 x^{21}+3 x^{22}+2 x^{23}+2 x^{24} \\
& +3 x^{25}+2 x^{26}+x^{27}+2 x^{28}+2 x^{29}+x^{30}+x^{32}+x^{33}+x^{36}
\end{aligned}
$$


Therefore, the minimum weighted dominating number of $G$ is 7 , and the coefficient of $x^{7}$ in $\mathcal{H}_{w}(x, \mathbf{x})$ given by $x_{3} x_{6}$ represents all the weighted dominating sets of weight equal to 7 .

\section{References}

[1] S. Akbari, S. Alikhani, and Y-H. Peng, "Characterization of graphs using domination polynomials", European journal of combinatorics, vol. 31, no. 7, pp. 1714-1724, 2010.

[2] S. Alikhani, Dominating sets and domination polynomials of graphs. Lap Lambert Academic , 2012.

[3] S. Alikhani and Y-H. Peng, "Introduction to domination poly nomial of a graph", Ars combinatoria, vol. 114, pp. 257-266, 2014.

[4] G. Chartrand, F. Harary and B. Quan Yue, "On the out- domination and in-domination numbers of a digraph", Discrete mathematics, vol. 197-198, pp. 179-183, 1999.

[5] J. Ghoshal, R. Laskar, and D. Pillone, "Topics on domination in directed graphs," in Domination in graphs advanced topics, T. W. Haynes, S. T. Hedetniemi, and P. J. Slater, Eds. New York, NY: Routledge, 1998, pp. 401-437.

[6] T. W. Haynes, S. T. Hedetniemi, and P. J. Slater, Fundamentals of domination in graphs. New York, NY: CRC, 1998.

[7] C. Pang, R. Zhang, Q. Zhang, and J. Wang, "Dominating sets in directed graphs", Information sciences, vol. 180, no. 19, pp. 3647-3652, 2010.

[8] F. Zou, Y. Wang, X-H. Xu, X. Li, H. Du, P. Wan, and W. Wu, "New approximations for minimum-weighted dominating sets and minimum-weighted connected dominating sets on unit disk graphs", Theoretical computer science, vol. 412, no. 3, pp. 198-208, 2011. 


\section{Jorge Alencar}

Instituto Federal de Educação, Ciência e Tecnolog a do Triângulo Mineiro Uberaba-MG,

Brazil

e-mail: jorgealencar@iftm.edu.br

Corresponding author

and

\section{Leonardo de Lima}

Departamento de Administração Geral e Aplicada

Universidade Federal do Paraná

Curitiba-PR,

Brazil

e-mail: leonardo.delima@ufpr.br 